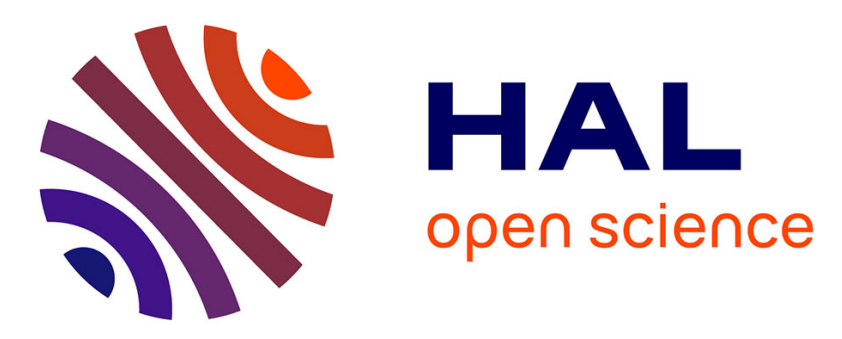

\title{
DNA Affects Ligand Binding of the Ecdysone Receptor of
}

Anca Azoitei, Margarethe Spindler-Barth

\section{To cite this version:}

Anca Azoitei, Margarethe Spindler-Barth. DNA Affects Ligand Binding of the Ecdysone Receptor of. Molecular and Cellular Endocrinology, 2009, 303 (1-2), pp.91. 10.1016/j.mce.2009.01.022 . hal00499113

\section{HAL Id: hal-00499113 https://hal.science/hal-00499113}

Submitted on 9 Jul 2010

HAL is a multi-disciplinary open access archive for the deposit and dissemination of scientific research documents, whether they are published or not. The documents may come from teaching and research institutions in France or abroad, or from public or private research centers.
L'archive ouverte pluridisciplinaire HAL, est destinée au dépôt et à la diffusion de documents scientifiques de niveau recherche, publiés ou non, émanant des établissements d'enseignement et de recherche français ou étrangers, des laboratoires publics ou privés. 


\section{Accepted Manuscript}

Title: DNA Affects Ligand Binding of the Ecdysone Receptor of Drosophila melanogaster

Authors: Anca Azoitei, Margarethe Spindler-Barth

PII: $\quad$ S0303-7207(09)00094-X

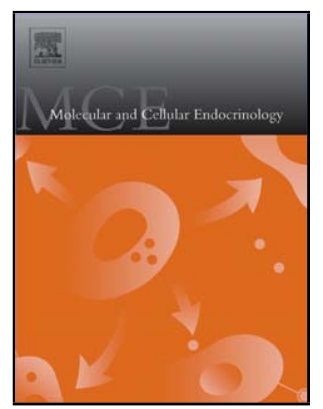

DOI: doi:10.1016/j.mce.2009.01.022

Reference: MCE 7136

To appear in: Molecular and Cellular Endocrinology

Received date:

$10-11-2008$

Revised date:

26-12-2008

Accepted date:

23-1-2009

Please cite this article as: Azoitei, A., Spindler-Barth, M., DNA Affects Ligand Binding of the Ecdysone Receptor of Drosophila melanogaster, Molecular and Cellular Endocrinology (2008), doi:10.1016/j.mce.2009.01.022

This is a PDF file of an unedited manuscript that has been accepted for publication. As a service to our customers we are providing this early version of the manuscript. The manuscript will undergo copyediting, typesetting, and review of the resulting proof before it is published in its final form. Please note that during the production process errors may be discovered which could affect the content, and all legal disclaimers that apply to the journal pertain. 
DNA Affects Ligand Binding of the Ecdysone Receptor of Drosophila melanogaster Anca Azoitei, Margarethe Spindler-Barth*

Institute of General Zoology and Endocrinology, University of UIm, Albert- Einstein allee 11, 89081 Ulm, Germany

${ }^{*}$ Corresponding author:

Prof. Dr. M. Spindler-Barth

Department of General Zoology and Endocrinology, University of UIm, 89069 UIm, Germany,

phone: +49 (0)7315022600,

fax : +49 (0)7315022581,

e - mail: margarethe.spindler-barth@uni-ulm.de 


\section{Summary}

The heterodimers of all three ecdysone receptor $(E c R)$ isoforms with Ultraspiracle (Usp), the invertebrate orthologue of RXR, bind ponasterone A with the same affinity in the absence of DNA. Ligand binding is stimulated by ecdysone response elements (EcREs) to different degrees depending on the receptor isoform, the heterodimerization partner, and the type of EcRE. Ligand binding to heterodimers with wtUsp is enhanced 5 fold with hsp27, Pal- 1 and DR-1. In the presence of DNA substantial differences in ligand binding were observed, when the $A B$ domain of wtUsp is replaced by the N-terminus of VP16, which is routinely used for determination of transcriptional activity to overcome the inhibitory action of the ABdomain of Usp. Enhanced dimerization in the presence of hormone response elements increases mainly the number of binding sites resulting in improved ligand binding, which is observed even if the C-domain of Usp is deleted. RXR, which can partially replace Usp function, confers high affinity ligand binding only in the presence of an EcRE.

Keywords: dimerization, hormone response element, insect, moulting hormone, nuclear receptor, retinoic acid receptor, ultraspiracle.

\section{Abbreviations used}

EcR: Ecdysone receptor, EcRE: ecdysone response element, Eos-FP: red fluorescent protein, Usp: Ultraspiracle, Vp16: herpes simplex transcriptional activator protein, RXR: Retinoic acid X-receptor, YFP: Yellow fluorescent protein. 


\section{Introduction}

Three isoforms of EcR with different AB-domains are described for Drosophila (Bender et al., 1997). "In vivo" these isoforms are not functionally equivalent and cannot substitute each other during Drosophila development. One possibility for finetuning of the hormone response in order to achieve isoform specific effects is variation of the expression level of receptor isoforms dependent upon the target tissue and developmental stage (Kamimura et al., 1997; Jindra et al. 1996; Verras et al., 2002). In addition the transcriptional capability of receptor isoforms varies particularly hormone induced transactivation. This has been shown for receptors expressed both in vertebrate (Beatty et al., 2006; Mouillet et al., 2001) and insect cells (Hu et al., 2003; Schubiger et al., 2003). Therefore the question arose, whether the various hormonal responses are due to differences in ligand binding affinity.

The ecdysteroid ponasterone A binds to the heterodimer EcR /Usp with high affinity (Yao et al., 1992). Since the affinity of separately expressed ligand binding domains are in the same range, when fused to Gal4-AD and Gal4-DBD respectively and the addition of other tags like GST, $\mathrm{His}_{6}$, or C-myc did not alter the affinity significantly (Grebe et al., 2004), it was concluded that the ligand binding domain is autonomous and that ligand binding is not affected by interaction with other receptor domains. On the other hand it is known from vertebrate nuclear receptors that interaction between the $\mathrm{AF}-1$ region in the $\mathrm{AB}$-domain and the ligand binding domain at the $\mathrm{C}$-terminus occurs either indirectly via comodulators (Tian et al., 2006; Ikonen et al., 1997) or directly (Tetel et al., 1999). Since the AB-domain is shaped into its active conformation by DNA (Lavery and McEwan, 2005) the interaction of AF-1 and AF-2 may require DNA binding of nuclear receptors as reported e.g. for the progesterone 
receptor by Heneghan et al. (2007). An influence of the ligand binding domains of EcR/Usp on the C-domain is reported e.g. DNA-binding of the receptor complex is enhanced severalfold in the presence of ligand (Antoniewski et al., 1993; Elke et al., 1999; Braun and Spindler-Barth, unpublished results). We therefore tested, whether the C-domain also affects ligand binding.

In most insects tested thus far, ligand binding to EcR is only detectable after heterodimerization. In Drosophila weak ligand binding is observed with EcR alone, but is reinforced about 90 fold by Usp (Grebe et al., 2003; Grebe et al., 2004). Usp increases the half life of the holo-receptor complex by reducing the dissociation rate of the ligand (Grebe et al., 2004). Mutational analysis revealed that Usp partially rescues the ligand binding capabilities of mutated EcRs (Grebe et al., 2003). We therefore examined, whether the influence of Usp is restricted to the E-domain, necessary for ligand induced dimerization, or whether other domains of Usp are also involved.

Several publications report that Usp can be replaced by the vertebrate orthologue RXR (Panguluri et al., 2006; Palli et al., 2005; Henrich et al., 2003; Thomas et al., 1993), albeit with lower efficiency (Nieva et al., 2008). We therefore examined the influence of RXR on ligand binding of the receptor heterodimer. 


\section{Material and Methods}

\subsection{Cell culture and transfection}

The Chinese hamster ovary (CHO-K1) cells were maintained in DMEM/F12 medium (with L-Glutamine) (Invitrogen, Carlsbad, Ca, USA) supplemented with 5\% FCS.

Cells were cultivated in tissue culture dishes at $37^{\circ} \mathrm{C}$ in a humidified atmosphere with $5 \% \mathrm{CO}_{2}$. For transfection $1.5-2 \times 10^{6}$ cells were seeded in $10 \mathrm{~cm}$ cell culture dishes. After $24 \mathrm{~h}(90 \%$ confluency) the transfection was performed with $7.0 \mu \mathrm{g}$ of DNA (3.5 $\mu \mathrm{g}$ DNA for each expression plasmid (EcR and Usp) using Lipofectamine 2000 (Invitrogen, Carlsbad, Germany) according to the prescription of the manufacturer. The cells were incubated for 24 hours before harvesting.

The following expression plasmids coding for nuclear receptors were used:

EcR isoforms: pcDNA3-dEcRA, pcDNA3-dEcRB1and pcDNA3-dEcRB2 was kindly provided by Dr.V.C. Henrich (University of Greensboro, NC, USA). The open reading frames were cloned between BamHI - Xbal sites in pcDNA3 vector (Invitrogen, Carlsbad, USA). The strategy of plasmid construction is described by (Mouillet et al., 2001).

Usp variants: Wild type Usp inserted into pEYFP-N1 (Clontech, Palo Alto, CA, USA) using EcoRI and Sall restriction sites was provided by Prof. Ozyhar (University of Wrozlav, Polen) and modified further by Dr. S. Braun (University of Ulm, Germany) to allow expression of a fusion protein encompassing aa 1-507 of Usp. DNA constructs coding for Usp variants (Uspl, Uspll and UsplII) were obtained from Dr. V.C. Henrich (University of North Carolina, Greensboro, USA). All Usp variants were cloned between EcoRI and HindIII into a pVP16 expression vector (Clontech, Heidelberg, 
Germany) replacing the A/B domain of Drosophila wild type Usp by VP16-AD (Beatty et al., 2006). The VP16-dUspl variant includes six amino acids of B domain of wild type Usp adjacent to the $\mathrm{N}$-terminus of the C- domain of Usp and the remaining carboxy-terminal part of the open reading frame (aa 98-507). The VP16-dUspll variant is identical to Uspl, with the exception that the six amino acids are missing. A third variant, VP16-dUspIII, coding for amino acids 170-507, lacks the DNA binding domain. $m R X R_{\alpha}$ was obtained from Dr. V.C. Henrich (University of North Carolina, Greensboro, USA) (Henrich et al., 2003).

\subsection{Preparation of cell extracts}

24 hours after transfection cells were gently detached from the surface with a scraper. The cells were rinsed with $5 \mathrm{ml}$ ice cold 1x PBS (138 mM NaCl, $2.7 \mathrm{~mm} \mathrm{KCl}$, $1.8 \mathrm{mM} \mathrm{KH}_{2} \mathrm{PO}_{4}, 10 \mathrm{mM} \mathrm{Na}_{2} \mathrm{HPO}_{4}, \mathrm{pH}=7.2$ ) and transferred into $15 \mathrm{ml}$ tubes. After centrifugation (260g, $4 \mathrm{~min}$ ) the supernatant was discarded and the cell pellet was resuspended in 3 packed volumes of ice cold extraction buffer (20 mM HEPES, 150 $\mathrm{mM} \mathrm{NaCl}, 1 \mathrm{mM}$ EDTA, $\mathrm{pH}=0$ 7.9) supplemented with $2 \mathrm{mM}$ DTT and protease inhibitors (aprotinin, leupeptin, pepstatin, $1 \mu \mathrm{g} / \mathrm{ml}$ each). After three freeze/thaw cycles the lysate was centrifuged $\left(18000 \mathrm{~g}, 30 \mathrm{~min}, 4^{\circ} \mathrm{C}\right)$. The clear supernatant was used for ligand binding assays. Protein content was determined according to Bradford (1949). Since the nuclear receptors especially Usp were not epressed at comparable levels (Braun et al., personal communication) separately expressed Usp was added to ensure that all EcR present was transferred into the heterodimer.

\subsection{Ligand binding}

Cell extracts prepared as described above were diluted with ligand binding dilution buffer (20 MM HEPES, $15 \%(\mathrm{v} / \mathrm{v})$ glycerol, 2 mM EDTA, 4 mM DTT, pH = 7.9) 
supplemented freshly with protease inhibitors (aprotinin, leupeptin, pepstatin, $1 \mu \mathrm{g} / \mathrm{ml}$ each). Ligand-binding was determined with ${ }^{3}[\mathrm{H}]$-Ponasterone A (specific activity 7.9 $\mathrm{TBq} / \mathrm{mmol}$ ) using a filter assay, as described previously (Turberg and Spindler, 1992). Briefly cell extracts were incubated routinely with $7.5 \mathrm{nM}^{3}[\mathrm{H}]-$ Ponasterone for $5 \mathrm{hrs}$ at $4^{\circ} \mathrm{C}$. For determination of $\mathrm{K}_{\mathrm{D}}$-values various concentrations of ${ }^{3}[\mathrm{H}]-$ Ponasterone A were used. Free ligand was removed by a filter assay (Turberg and Spindler, 1992) using NC45 nitrocellulose membranes (Schleicher and Schuell, Dassel, Germany) washed with 3 x $4 \mathrm{ml}$ ice-cold washing buffer (20mM HEPES, 20mM NaCl, 10\%glycerol (v/v), 1 mM EDTA, 1 mM ß-mercaptoethanol, $\mathrm{pH}=7.9$ ). Bound radioactivity was measured with a liquid scintillation counter (TRicarb 1500, Perkin Elmer, Rodgau-Jügesheim, Germany) and the non specific binding

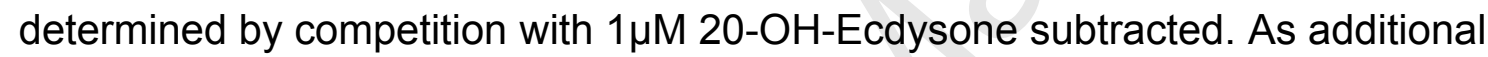
control extracts from non transfected cells were used $(<100 \mathrm{cpm})$. The purity of ${ }^{3}[\mathrm{H}]-$ Ponasterone A was routinely checked by HPLC analysis before use. For calculation of $K_{D}-$ values the results were evaluated according to Scatchard (1949) or calculated with Kaleida Graph, version 4.0 (Synergy Software, Reading, PA, USA). Comparable results were obtained with both methods; each experiment was repeated 3-6 fold.

The influence of different hormone response elements (280 ng / 100 $\mu$ l) was studied using the same receptor concentration for each set of experiments. The amount of receptor was determined by Scatchard analysis/Kaleidagraph. The following double stranded oligonucleotides were used.

1). 1x hsp 27: AGCGACAAGGGTTCAATGCACTTGT ATTGGACAAGTGCATTGAACCCTTGT

2). 3x hsp 27: (AGCGACAAGGGTTCAATGCACTTGT 
ATTGGACAAGTGCATTGAACCCTTGT) $)_{3}$

3). 5x hsp 27: (AGCGACAAGGGTTCAATGCACTTGT

ATTGGACAAGTGCATTGAACCCTTGT) $)_{5}$

4).1x DR-1: GATCTAGAGAGGTCAAAGGTCATGTCCAAG

GATCCTTGGACATGACCTTTGACCTCTCTA

5). 5xDR-1 (GATCTAGAGAGGTCAAAGGTCATGTCCAAG

GATCCTTGGACATGACCTTTGACCTCTCTA) 5

6).1XPal-1: GATCTAGAGAGGTCAATGACCTCGTCCAAG

GATCCTTGGACGAGGTCATTGACCTCTCTA

7). 5xPal-1: (GATCTAGAGAGGTCAATGACCTCGTCCAAG

GATCCTTGGACGAGGTCATTGACCTCTCTA) 5

Hsp 27 was purchased from Thermo (Ulm, Germany) and Pal-1 and DR-1 were obtained from Biomers (Ulm, Germany). Controls with the same amount of nonspecific DNA of comparable size were performed and subtracted $(<150 \mathrm{cpm})$.

Statistical significance ( $P$ values less than 0.01 ) of differences among groups was determined on the basis of a two-sided, matched paired Student's $t$ test. 


\section{Results}

3. 1. Ligand binding to EcR isoforms in the absence of Usp is reinforced by DNA. Ligand binding affinity of EcR is about 90 fold lower in the absence of a heterodimerization partner as determined by kinetic measurements of receptor domains expressed in yeast cells (Grebe et al., 2004). Control experiments revealed that during the time period needed for the experiments ( 5 hours) the receptor proteins remained functional concerning ligand binding even in the absence of hormone and /or dimerization partner. After ten hours $93.5 \pm 3.5 \%(n=6)$ of the ligand binding capacity of the receptor protein of each isoform is still present. We therefore conclude that no degradation occurred during the incubation time in the extracts. In the presence of the ecdysone response element (EcRE) DR-1 ligand binding is improved particulary if oligomeric EcREs are used (Fig. 1). The stimulatory effect varies depending on the EcR isoform and is significant only for EcR-A and EcR-B1, but not for EcR-B2 and indicates a modulatory effect of the AB domain of EcR. We can not discriminate, whether ligand binding is improved by an increase in homodimerization or whether there is a direct influence of the DBD complexed with the hormone response element on ligand binding. Determination of ligand binding revealed a $K_{D}=$ $3.3 \times 10^{-8} \mathrm{M} / \mathrm{L}$ for EcR-A in the presence of DR-1 (Fig 2). Our efforts to determine $\mathrm{K}_{\mathrm{D}}$-values in the absence of DNA were not successful under our experimental conditions, although at a concentration of $10 \mathrm{nM} / \mathrm{L}{ }^{3} \mathrm{H}$-ponasterone A specific ligand binding above background level (100-150cpm) could be detected (Fig.1).

\subsection{All three isoforms of Drosophila EcR bind ligand with the same affinity, if complexed with Usp.}


The affinity of Ponasterone A (Table 1, Fig. 3) is the same for heterodimers of all three isoforms of EcR and wild type Usp in the absence of DNA. Since the ABdomain of Usp is routinely replaced by the N-terminal activation domain of the $\mathrm{Vp} 16$ transcription factor in order to overcome the inhibitory influence of the original Usp $\mathrm{A}_{\mathrm{AB}}$ domain on transcriptional activity (Henrich, 2005), we additionally determined ligand affinity of the heterodimers with $V p 16_{A D}-U s p_{C D E}$ fusion proteins (Uspl and II). No significant influence of the AB- domain of Usp on ligand binding was observed (Table 1). Deletion of the DNA binding domain of Usp, which also harbours a strong dimerization interface, does not affect ligand binding (Table 1). Fusion of EcR with a fluorescent protein such as YFP at the $\mathrm{N}$-terminal end also does not impair ligand binding and the $K_{D}$-values are only slightly increased $(10.7 \% \pm 3.2 \%, n=6)$. The number of binding sites varies about 6 fold (table 1), which is not due to degradation processes in the extract, since about $96 \%$ of the ligand binding capacity is still present 5 hours after the experiments were performed.

\subsection{Ligand binding of ecdysone receptor heterodimers with Usp is increased in the} presence of DNA

To investigate whether interaction with DNA modulates ligand binding to EcR/Usp, we performed hormone-binding experiments in the presence of hormone response elements. Receptor concentrations in the absence of DNA were determined for each cell extract by Scatchard plot/Kaleidagraph to ensure that the same amount of ligand binding sites were used in each experiment. Monomers of the ecdysone response element hsp27, which are routinely used for DNA binding studies such as gel mobility shift assays, increase ligand binding to all EcR isoforms in the presence of wild type Usp about 4-6 fold (Fig 4A). For determination of transcriptional activity tandemly 
arranged EcREs oligomers were often used to enhance the hormonal response (Henrich, 2005). Therefore hormone response element oligomers were also tested (Fig. 4). No difference between EcRE monomers and oligomers was found with all heterodimers consisting of all EcR isoforms and wild type Usp.

3.4. In the presence of DNA the AB domain of Usp modifies ligand binding to EcR. In transcriptional assays the AB-domain of Usp is replaced routinely by the $\mathrm{N}$ terminus of Vp16 (Henrich, 2005) as mentioned above. In this case pronounced differences in ligand binding depending on the EcR isoform and the type of EcRE present are obtained (Fig. 5). In contrast to 1x hsp 27, Pal-1 and DR-1 monomers can enhance ligand binding in some circumstances e.g. for EcR-B1/Uspl. The stimulatory effect of EcRE pentamers is less pronounced in case of EcR-A. A similar result is obtained with Uspll, which lacks the hexapeptide of the original wtUsp adjacent to the $\mathrm{N}$-terminus of the C-domain. (Fig. 6).

To investigate, whether the increase in ligand binding is due to altered ligand affinity or increased number of binding sites due to enhanced heterodimerization in the presence of DNA we determined $K_{D}$-values and receptor concentrations for some selected receptor complexes. As shown for EcR-B1/Uspll in the presence of 5xDR-1 (Fig. 7A and $B$ ), the number of binding sites increases in the presence of this EcRE from $0.19 \pm 0.024 \mathrm{nM} / \mathrm{L}$ in the absence of DNA to $1.14 \pm 0.28 \mathrm{nM} / \mathrm{l}$ in the presence of $5 \times D R 1$, but simultaneously the affinity of the ligand is slightly impaired (about 2fold in the presence of $5 \times D R 1$ as shown in Fig. 7. Comparable results were obtained also with heterodimers with other Usp variants and EcR-B1 (data not shown). 
3.5. Deletion of the DNA binding domain of Usp improves ligand binding to EcR in the presence of DNA in an isoform specific manner.

As outlined previously in the absence of DNA ligand binding of cell extracts is not changed, if the C-domain of Usp is deleted and no influence of the EcR isoform is detectable (Table 1). However, in the presence of EcREs, deletion of the DNA binding domain of Usp selectively stimulates ligand binding. This effect is considerably higher in heterodimers with EcR-A compared to EcR-B1 and EcR-B2 both in the presence of DR-1 and PAL-1 monomers and pentamers (Fig. 8, for comparison see Fig. 5 and 6$)$.

3.6. Enhanced ligand binding in the presence of DNA is mainly due to improved dimerization.

To investigate, whether the increase in ligand binding is due to altered ligand affinity or increased number of binding sites due to enhanced heterodimerization in the presence of DNA we determined $K_{D}$-values and receptor concentrations for some selected receptor complexes. As shown for EcR-B1/Uspll in the presence of 5xDR-1 (Fig. 7A and B), the number of binding sites increases in the presence of the EcRE, but simultaneously the affinity of the ligand is slightly impaired. Comparable results were obtained also with heterodimers with other Usp variants and EcR-B1 (data not shown). Even in the absence of the DNA-binding domain of Usp (heterodimers with UspIII) the number of binding sites is increased in the presence of the EcRE. (Fig. 9) Since the same cell extracts were used for determination of ligand binding with and without DNA, it is reasonable to assume that the number of heterodimeric receptor 
complexes increased in the presence of a suited EcRE due to increased dimerization of the receptor molecules.

3.7. $R X R$ can partially replace Usp in ligand binding assays only in the presence of DNA

If Usp is replaced by $R X R$, no high affinity hormone binding is observed (data not shown). However, in the presence of a hormone response element, RXR also confers ligand binding to the heterodimer (Fig. 10). Ligand affinity is in a comparable range compared to heterodimers with Usp, but the number of binding sites is reduced (concentration of EcR-B1/RXR = $0.07 \mathrm{nM} / \mathrm{L}$ compared to EcR/Usp concentration = $1.05 \mathrm{nM} / \mathrm{L}$ (see fig.7) in the presence of 5xDR1) indicating that heterodimerization of EcR with RXR is less efficient compared to formation of EcR/Usp complexes. 


\section{Discussion}

The influence of hormone on nuclear receptor function is studied mainly by transcriptional assays. However ligand induced changes in receptor function are the result of a variety of different receptor properties e.g. dimerization and DNA binding of the receptor protein. Hormone and/or heterodimerization also enhance the concentration of the expressed receptor proteins (Ruff, unpublished results) in an isoform specific manner. Direct measurements of ligand binding allow evaluation of the impact of different receptor domains, DNA and dimerization partner on hormoneinduced effects.

\subsection{Interaction of EcR with DNA improves ligand binding in the absence of a heterodimerization partner.}

Unpartnered EcR from Drosophila melanogaster like that from Leptinotarsa decemlineata (Ogura et al., 2005) binds ponasterone A specifically, although with lower ligand affinity than the heterodimeric ecdysone receptor (Grebe et al., 2003). Since ligand affinity to cell extracts containing only EcR is about 90 fold lower compared to heterodimers with Usp, the biological significance of hormone binding of unpartnered EcR was often considered to be neglectable, even though ligand induced changes in receptor function in the absence of a heterodimerization partner had been demonstrated repeatedly e.g. enhanced interaction with DNA (Braun, personal communication) and chromatin (Cronauer et al., 2007). Recently Constantino et al. (2008) showed that ecdysone signalling is mediated in a certain developmental stage without participation of Usp or any other RXR like molecule. Transcriptional activity is also stimulated in the presence of Muristerone A in 
vertebrate cells transfected with EcR to different degrees depending on the isoform used (Beatty et al., 2006; Tremmel and Ruff, unpublished observations). This is not due to interaction with endogenous RXR, since the subclone of $\mathrm{CHO}-\mathrm{K} 1$ used in our lab expresses only neglectable amounts of RXR. Even after cotransfection with plasmids coding for RXR only hormone induced transcriptional activity but not ligand binding in cell extracts in the absence of DNA is enhanced (Nieva et al., 2008). It is important to determine the RXR content of the cells used for these experiments, because the RXR content may vary depending on the subclone of $\mathrm{CHO}-\mathrm{K} 1$ cells. For example constitutively expressed $\mathrm{RXR}$ in $\mathrm{CHO}$-cells is reported by Hannan and Hill (1997).

The increase in ligand binding in the presence of an EcRE (Fig. 1) shows that not only dimerization via the ligand binding domains, but also via C-domains in the presence of DNA positively affects the ligand binding domain of EcR presumably by stimulating homodimerization mediated by the strong dimerization interface in the DNA binding domain. Comparison with data reported by Grebe et al. (2004) revealed that ligand binding is enhanced about 3-4-fold in the presence of DNA, but is still about 20-25 fold lower compared to ligand affinity of the heterodimer.

\subsection{The $A B$ - domain of EcR does not affect ligand binding of EcR/Usp in the absence, but in the presence of DNA.}

Hormone binding of unpartnered EcR varies in the absence or presence of DNA and depending on the $A B$ domains of the receptor isoforms (Fig. 1). In contrast ligand affinity of heterodimers with Usp is comparable for all EcR isoforms in the absence of DNA. Similar results were also reported by Perera et al. (1999) for the ecdysone 
receptor of Choristoneura fumiferana. The affinity of EcR/Usp is in accordance with the data reported previously for heterodimers with the EcR-B1 isoform (Yao et al., 1992) as well as with heterodimers encompassing only the ligand binding domains of EcR and Usp fused to Gal4-AD and Gal4-DBD respectively (Grebe et al., 2003). Therefore, it was concluded that ligand binding of EcR/Usp is not influenced by other domains of the ecdysone receptor molecule. This is in agreement with experiments, which show that various tags at the $\mathrm{N}$-terminal end do not affect ligand binding (Grebe et al., 2003; this paper) and hormonal stimulation of transcriptional activity is only slightly impaired (Nieva et al., 2005).

However in the presence of DNA the AB-domain of EcR isoforms affects ligand binding of heterodimers (Figures 4-8). Since binding of nuclear receptors to DNA results in allosteric interaction between DBD and N-terminal regions it was expected that the $A B$ domain of EcR affects dimerization mediated by the C-domains, thus modifiying the number of heterodimers, but ligand affinity is also slightly impaired indicating a more complex interaction of the receptor domains. An influence of the AB-domain of nuclear receptors on ligand induced transcriptional activity has been reported a number of times for different isoforms of vertebrate nuclear receptors (Tian et al., 2006; Matthews and Gustafsson, 2003; Bevan et al., 1999) due to interaction of the $\mathrm{N}$-terminus with the ligand binding domain (Takimoto et al., 2003). Isoform specific interaction of $\mathrm{N}$ - and $\mathrm{C}$-terminus of EcR was shown by FRET analysis (Tremmel and Schaefer, unpublished results). At present it is not clear, whether this interaction may also affect ligand binding.

\subsection{Influence of the $A B$ domain of the heterodimerization partner on ligand binding in} the presence of DNA. 
Although ligand binding is stimulated in the presence of DNA there was no observed influence of the type of EcRE or the AB-domain of EcR on ligand binding to EcR/Usp heterodimers. However, this is true only if the original $A B$ domain of wild type Usp is maintained (Fig. 4). Because transcriptional activity of heterodimers with wild type Usp and in particular to EcR-B1 is rather low even in the presence of hormone, the AB-domain is routinely replaced by the N-terminus of VP16 (Henrich, 2005). Surprisingly this exchange has considerable consequences for ligand binding to the heterodimer in the presence of DNA (Fig. 5-6). These results show that Usp is not simply a passive partner as has been previously proposed (Hu et al., 2003), but plays an active role in receptor mediated hormone action. The results presented here further demonstrate that replacement of the original AB-domain of wild type Usp alters transcriptional activity of the heterodimer not only qualitatively but also quantitatively. In contrast to the heterodimers of all EcR isoforms and wild type Usp, receptor complexes with $\mathrm{Vp} 16$ - Usp fusion proteins modify ligand binding of the heterodimers in a complex way, dependent upon the EcR isoform and the type of EcRE. This means that the physiological consequences of isoform specific hormone effects obtained in the presence of VP16-Usp fusion proteins should be interpreted cautiously and do not necessarily reflect the physiological situation.

\subsection{Deletion of the DNA binding domain of Usp selectively modifies ligand binding in} the presence of DNA

According to Niedziela-Majka et al. (2000), the C-domain of Usp has a profound influence on DNA binding of the receptor complex directing EcR to the 5'end of the hormone response element. Nevertheless heterodimerization via interaction of the ligand binding domains is still observed according to gel mobility shift assays (Beatty 
et al., 2006). Therefore we expected that deletion of the DNA binding domain of Usp would also modify the impact of DNA binding on ligand affinity to the receptor complexes.

Although dimerization is considerably weaker in the absence of the C-domain of Usp (Azoitei et al., unpublished observation), ligand binding in the absence of DNA is not changed when compared to heterodimers with full length Usp's, as mentioned above (Table 1). Since the stimulation of ligand binding by addition of EcREs is even more pronounced in the absence of the dimerization interface of the C-domain of Usp (compare Figures 4-6 with Fig. 8 ), the type of dimerization, which can be mediated only by the E-domain of both nuclear receptors obviously allows a more efficient ligand binding.

\subsection{RXR confers ligand binding to EcR only in the presence of DNA.}

In the presence of a strong heterodimerization partner like Usp, dimerization via the ligand binding domains seems to be sufficient to confer high affinity ligand binding to EcR even in the absence of DNA, since ligand affinity is not impaired if the C-domain of Usp is deleted. If only weak dimerization mediated by the ligand binding domains is possible as is the case with EcR homodimers or heterodimers with RXR, dimerization via the C-domains, which does not seem possible in solution (Rastinejad et al., 1995) but is reinforced in the presence of a suited EcRE, allows still high affinity ligand binding (Fig. 10).

Our results explain why RXR can replace Usp in transcriptional assays (Panguluri et al., 2006; Palli et al., 2005; Henrich et al., 2003; Thomas et al., 1993) but not in other 
functional tests, which do not require interaction with DNA such as nuclear import (Nieva et al., 2008).

4.6 Physiological consequences of modulation of ligand binding by DNA and dimerization partners

The intense intra- and intermolecular interactions described here, which allow modulation of ligand binding by the $\mathrm{AB}$ - and $\mathrm{C}$-domains of the receptor molecule, the type and number of EcREs and dimerization partners, provide further examples of the high flexibility of the nuclear receptor complexes such as the ecdysone receptor. This in turn offers multiple possibilities for fine-tuning of the hormonal response. As outlined previously, receptor domains like the ligand binding domain (Billas et al., 2003) or the C-domain (Orlowski et al., 2004) are rather flexible, which certainly facilitates modification of receptor activity. 


\section{Acknowledgements}

${ }^{3} \mathrm{H}$ - Ponasterone A was a kind gift of Dr. H. Kayser (Syngenta, Basel, Switzerland). A plasmid coding for wild type Usp was kindly provided by Dr. S. Braun, who modified a precursor plasmid obtained by Dr. Ozyhar (Technical University of Wroclav, Poland) to allow expression of wild type Usp. Expression plasmids for EcR-A, EcR-B1, EcRB2 and Usp I, II and III and RXR, were kindly provided by Dr. V.C. Henrich (University of North Carolina, Greensboro, NC, USA). Antibody AB11 was a gift of Dr. Kafatos (EMBL, Heidelberg, Germany). The monoclonal antibody Ag10.2 developed by Thummel and Hogness was obtained from the Developmental Studies Hybridoma Bank developed under the auspices of the NICHD and maintained by the University of lowa, Department of Biology, lowa City, IA 55242 USA. Ponasterone A was purified by M. Burret (University of Ulm). We kindly acknowledge also his help by preparing the figures. The work was supported by a grant from the DFG to MSB (Spi 350/5-1 and 2) and a PhD-scholarship to AA (Graduate college 1041). The technical assistance of N. Möbius, the help of K. Belisle, who corrected and improved the English version of the manuscript is gratefully acknowledged. 


\section{Legends}

Fig.1: Influence of the hormone response element 5x DR1 on ligand binding to EcR isoforms in the absence of a heterodimerization partner. White bars = no DNA present, black bars $=$ addition of 280 ng $5 \times$ DR $1 / 100 \mu l$. The differences between bars indicated by *, ** and ${ }^{+}$are significant $(p<0.01, n=3) .10 \mathrm{nM} / \mathrm{L}^{3} \mathrm{H}$-ponasterone $\mathrm{A}$ (spec. activity: $7.9 \mathrm{TBq} / \mathrm{mmol}$ )) was used. Non specific binding was subtracted as described in material and methods.

Fig. 2: Ligand binding of EcR-B1 (transfected in CHO-K1-cells) in the presence of the hormone response element 5x DR-1. ${ }^{3} \mathrm{H}-$ Ponasterone (specific activity: $7.9 \mathrm{TBq} /$ $\mathrm{mmol}$ ) was used as ligand ( $n=4$, one representative experiment is shown). Data are analysed according to Scatchard (1949) and Kaleida Graph (inset). $\mathrm{K}_{\mathrm{D}}=33.9 \mathrm{nM} / \mathrm{L}$ receptor concentration $=0.11 \mathrm{nM} / \mathrm{L}$ (calculated with Kaleidagraph),

Fig. 3: Ligand binding of heterodimers of EcR isoforms and wild type Usp cotransfected in $\mathrm{CHO}-1 \mathrm{~K}$ cells ( $n=5$, one representative experiment is shown). Data are analysed according to Scatchard (1949) and Kaleida Graph (inset). A) EcR-A, B) EcR-B1, C) EcR-B2. $K_{D}$-values and receptor concentrations see table 1.

Fig. 4: Influence of hormone response elements on ligand binding (Ponasterone A) to heterodimers of EcR isoforms with wild type Usp. Binding experiments were performed with cell extracts containing $10 \mathrm{nM}$ receptor complex according to Scatchard / Kaleida Graph analysis. (Mean \pm SD, $n=3$ ). A) Ponasterone A binding in presence of $1 \mathrm{x}$ hsp27 and 5x hsp27. B) Ligand binding in presence of 1x PAL-1 and 5x PAL-1. C) Ponasterone A binding in the presence of $1 \times$ DR-1 and 5x DR-1. Black 
bars: Ligand binding in the absence of DNA, hatched bars: Ligand binding in the presence of hre monomers, white bars: Ligand binding in the presence of EcRE pentamers. The differences between bars indicated by ${ }^{*},{ }^{* *}$ and ${ }^{+}$are significant $(p<0.01, n=3)$. Non specific binding was subtracted as described in material and methods.

Fig. 5: Influence of hormone response elements on ligand binding (Ponasterone A) to heterodimers of EcR isoforms with Uspl. Binding experiments were performed with cell extracts containing $10 \mathrm{nM}$ receptor complex according to Scatchard /(Kaleida Graph analysis. A) Ponasterone A binding in presence of $1 \mathrm{x}$ hsp27 and $5 \mathrm{x}$ hsp27. $($ Mean $\pm S D, n=3)$ B) Ligand binding in presence of $1 \times$ PAL-1 and $5 \times$ PAL-1. C) Ponasterone A binding in the presence of $1 \times$ DR-1 and 5x DR-1. Black bars: Ligand binding in the absence of DNA, hatched bars: Ligand binding in the presence of hre monomers, white bars: Ligand binding in the presence of EcRE pentamers. The differences between bars indicated by ${ }^{*},{ }^{* *}$ and ${ }^{+}$are significant $(p<0.01, n=3)$. Non specific binding was subtracted as described in material and methods.

Fig. 6: Influence of hormone response elements on ligand binding (Ponasterone A) to heterodimers of EcR isoforms with Usp II. Binding experiments were performed with cell extracts containing 10nM receptor complex according to Scatchard/(Kaleida Graph analysis (Mean $\pm S D, n=3$ ). A) Ponasterone A binding in presence of $1 x$ hsp27 and 5x hsp27. B) Ligand binding in presence of 1x PAL-1 and 5x PAL-1. C) Ponasterone A binding in the presence of $1 \times$ DR-1 and 5x DR-1. Black bars: Ligand binding in the absence of DNA, hatched bars: Ligand binding in the presence of EcRE monomers, white bars: Ligand binding in the presence of EcRE pentamers. 
The differences between bars indicated by ${ }^{*},{ }^{* *}$ and ${ }^{+}$are significant $(p<0.01, n=3)$. Non specific binding was subtracted as described in material and methods.

Fig. 7: Ligand binding to EcR-B1/Uspll cotransfected in $\mathrm{CHO}$ cells in the presence of 5x DR1. ${ }^{3} \mathrm{H}$-Ponasterone (specific activity $7.9 \mathrm{~Bq} / \mathrm{mmol}$ ) was used as ligand. A) In the absence of DNA, $K_{D}$-value $=3.82 \mathrm{nM} / \mathrm{L}$, receptor concentration: $0.20 \mathrm{nM} / \mathrm{L} B$ ) In the presence of $5 \times \mathrm{DR} 1, \mathrm{~K}_{\mathrm{D}}$-value $=8.4 \mathrm{nM} / \mathrm{L}$, receptor concentration: $1.05 \mathrm{nM} / \mathrm{L}$. Data are analysed according to Scatchard (1949) and Kaleida Graph (inset). ( $n=3$, one representative experiment is shown).

Fig. 8: Influence of hormone response elements on ligand binding (Ponasterone A) to heterodimers of EcR isoforms with Usplll. Binding experiments were performed with cell extracts containing $10 \mathrm{nM}$ receptor complex according to Scatchard /(Kaleida Graph analysis (Mean $\pm S D, n=3$ ). A) Ponasterone A binding in presence of $1 x$ hsp27 and 5x hsp27. B) Ligand binding in presence of 1x PAL-1 and 5x PAL-1. C) Ponasterone A binding in the presence of $1 \times$ DR-1 and 5x DR-1. Black bars: Ligand binding in the absence of DNA, hatched bars: Ligand binding in the presence of EcRE monomers, white bars: Ligand binding in the presence of EcRE pentamers.

Fig. 9: Ligand binding to EcR-A/Usplll cotransfected in $\mathrm{CHO}$ cells. ${ }^{3} \mathrm{H}-\mathrm{Ponasterone}$ (specific activity $7.9 \mathrm{~Bq} / \mathrm{mmol}$ ) was used as ligand. Data are analysed according to Scatchard (1949) and Kaleida Graph (inset). ( $n=3$, one representative experiment is shown). A) In the absence of DNA, $K_{D}-$ value $=4.17 \mathrm{nM} / \mathrm{L}$, receptor concentration $=$ $0.21 \mathrm{nM} / \mathrm{L} \mathrm{B}$ ) in the presence of $5 \times \mathrm{DR} 1, \mathrm{~K}_{\mathrm{D}}-$ value $=7.1 \mathrm{nM} / \mathrm{L}$, receptor concentration: $1.46 \mathrm{nM} / \mathrm{L}$. 
Fig. 10: Ligand binding of heterodimers of EcR - B1 and RXR cotransfected in CHO$1 \mathrm{~K}$ cells in the presence of $5 \mathrm{x}$ DR1. ${ }^{3} \mathrm{H}$-Ponasterone (specific activity: $7.9 \mathrm{TBq} /$ mmol) was used as ligand. Data are analysed according to Scatchard (1949) and Kaleida Graph (inset). ( $n=6$, one representative experiment is shown). $K_{D}$-value $=$ $7.29 \mathrm{nM} / \mathrm{L}$, receptor concentration= $0.07 \mathrm{nM} / \mathrm{L}$. 


\section{Literature}

Antoniewski, C., Laval, M., Lepesant, J.A., 1993. Structural features critical to the activity of an ecdysone receptor binding site. Insect Biochem. Mol. Biol. 23,105-114.

Beatty, J., Fauth, Th., Callendar, J.L., Spindler-Barth, M., Henrich, V.C., 2006.

Functional analysis of ecdysteroid receptor isoforms in Drosophila melanogaster in a cell culture system. Insect Mol. Biol. 15, 785-795.

Bender, M., Imam, F.B., Talbot, W.S., Ganetzky, B., Hogness, D.S., 1997. Drosophila ecdysone receptor mutations reveal functional differences among receptor isoforms. Cell 91, 777-788.

Bevan, C.L., Hoare, S., Claessens, F., Heery, D.M., Parker, M.G., 1999. The AF1 and AF2 domains of the androgen receptor interact with distinct regions of SRC1. Mol. Cell. 19, 8383-8392.

Billas, I.M., Iwema, T., Garnier, J.M., Mitschler, A., Rochel, N., Moras, D., 2003. Structural adaptability in the ligand-binding pocket of the ecdysone hormone receptor. Nature 426, 91-96. 
Bradford, M.M., 1976. A rapid and sensitive method for the quantitation of microgram quantities of protein utilizing the principle of protein-dye interaction. Anal. Biochem. $72,248-254$.

Costantino, B.F., Bricker, D.K., Alexandre, K., Shen, K., Merriam, J.R., Antoniewski, C., Callender, J.L., Henrich, V.C., Presente, A., Andres, A.J., 2008. A novel ecdysone receptor mediates steroid-regulated developmental events during the mid-third instar of Drosophila.PLoS Genet. 4, e1000102.

Cronauer, M.V., Braun, S., Tremmel, Ch., Kröncke, K.-D., Spindler-Barth, M., 2007. Nuclear localization and DNA binding of ecdysone receptor and ultraspiracle. Arch. Insect Biochem. Physiol. 65,125-133.

Elke, C., Rauch, P., Spindler-Barth, M., Spindler, K.-D., 1999. DNA-binding properties of the ecdysteroid receptor-complex (EcR/USP) of the epithelial cell line from Chironomus tentans. Arch. Insect Biochem. Physiol. 41, 124-133.

Grebe, M., Przibilla, S., Henrich, V.C., Spindler-Barth, M., 2003. Characterization of the ligand-binding domain of the ecdysteroid receptor from Drosophila melanogaster. Biol. Chem. 384,105-116.

Grebe, M., Fauth, T., Spindler-Barth, M., 2004. Dynamic of ligand binding to Drosophila melanogaster ecdysteroid receptor. Insect Biochem. Mol. Biol. 34, 981989. 
Hannan, G.N., Hill, R.J., 1997. Cloning and characterization of LcEcR: a functional ecdysone receptor from the sheep blowfly Lucilia cuprina. Insect Biochem. Mol. Biol. $27,479-488$.

Heneghan, A.F., Connaghan-Jones, K.D., Miura, M.T., Bain, D.L., 2007. Coactivator assembly at the promoter: efficient recruitment of SRC2 is coupled to cooperative DNA binding by the progesterone receptor. Biochemistry 46, 11023-11032.

Henrich, V.C., 2005. The ecdysteroid receptor. In: Gilbert L.J., latrou K. (eds.) Comprehensive Molecular Insect Science. Elsevier, Pergamon, Oxford, Vol. 3, pp. 243-282.

Henrich, V.C., Burns, E., Yelverton, D.P., Christensen, E., Weinberger, C., 2003. Juvenile hormone potentiates ecdysone receptor-dependent transcription in a mammalian cell culture system. Insect Biochem. Mol. Biol. 33, 1239-1244.

Hu, X., Cherbas, L., Cherbas, P., 2003. Transcription activation by the ecdysone receptor (EcR/USP): identification of activation functions. Mol. Endocrinol. 17, 716731.

Ikonen, T., Palvimo, J.J,, Jänne, O.A., 1997. Interaction between the amino- and carboxyl-terminal regions of the rat androgen receptor modulates transcriptional activity and is influenced by nuclear receptor coactivators. J. Biol. Chem. 272, 2982129828. 
Jindra, M., Malone, F., Hiruma, K., Riddiford, L.M., 1996. Developmental profiles and ecdysteroid regulation of the mRNAs for two ecdysone receptor isoforms in the epidermis and wings of the tobacco hornworm, Manduca sexta. Dev. Biol. 180, 258272.

Kamimura, M., Tomita, S., Kiuchi, M., Fujiwara, H., 1997. Tissue-specific and stagespecific expression of two silkworm ecdysone receptor isoforms - ecdysteroiddependent transcription in cultured anterior silk glands. Eur. J. Biochem. 248, 786-93.

Lavery, D.N., McEwan, I.J., 2005._Structure and function of steroid receptor AF1 transactivation domains: induction of active conformations. Biochem. J. 391, 449464.

Lämmli, U.K., 1970. Cleavage of structural proteins during the assembly of the head of bacteriophage T4. Nature 27, 680-685.

Lavery, D.N., McEwan, I.J., 2005. Structure and function of steroid receptor AF1 transactivation domains: induction of active conformations. Biochem. J. 391, 449464.

Matthews, J., Gustafsson, J.A., 2003. Estrogen signaling: a subtle balance between ER alpha and ER beta. Mol. Interv. 3, 281-292.

Mouillet, J.F., Henrich, V.C., Lezzi, M., Vögtli, M., 2001. Differential control of gene activity by isoforms A, B1 and B2 of the Drosophila ecdysone receptor. Eur. J. Biochem. 268, 1811-1819. 
Niedziela-Majka, A., Kochman, M., Ozyhar, A., 2000. Polarity of the ecdysone receptor complex interaction with the palindromic response element from the hsp27 gene promoter. Eur. J. Biochem. 267, 507-519.

Nieva, C., Gwóźdź, T., Dutko-Gwóźdź, J., Wiedenmann, J., Spindler-Barth, M., Wieczorek, E., Dobrucki, J., Duś, D., Henrich, V., Ozyhar, A., Spindler, K.-D., 2005. Ultraspiracle promotes the nuclear localization of ecdysteroid receptor in mammalian cells. Biol. Chem. 386, 463-470.

Nieva, C., Spindler-Barth, M., Spindler, K.-D. 2008. Impact of heterodimerization on intracellular localization of the ecdysteroid receptor (EcR). Arch. Insect Biochem. Physiol. 68, 40-49.

Ogura, T., Minakuchi, C., Nakagawa, Y., Smagghe, G., Miyagawa, H., 2005. Molecular cloning, expression analysis and functional confirmation of ecdysone receptor and ultraspiracle from the Colorado potato beetle Leptinotarsa decemlineata. FEBS J. 272, 4114-4128.

Orlowski, M., Szyszka, M., Kowalska, A., Grad I, Zoglowek, A., Rymarczyk, G., Dobryszycki, P., Krowarsch, D., Rastinejad, F., Kochman, M., Ozyhar, A., 2004. Plasticity of the ecdysone receptor DNA binding domain. Mol. Endocrinol. 18, 21662184.

Palli, S.R., Kapitskaya, M.Z., Potter, D.W., 2005. The influence of heterodimer partner ultraspiracle/retinoid $\mathrm{X}$ receptor on the function of ecdysone receptor. FEBS J. $272,5979-5979$. 
Panguluri, S.K., Kumar, P., Palli, S.R., 2006. Functional characterization of ecdysone receptor gene switches in mammalian cells. FEBS J. 273, 5550-5563.

Perera, S.C., Ladd, T.R., Dhadialla, T.S., Krell, P.J., Sohi, S.S., Retnakaran, A., Palli, S.R., 1999. Studies on two ecdysone receptor isoforms of the spruce budworm, Choristoneura fumiferana. Mol. Cell. Endocrinol. 152, 73-84.

Rastinejad, F., Perlmann, T., Evans, R.M., Sigler, P.B., 1995. Structural determinants of nuclear receptor assembly on DNA direct repeats. Nature 375, 190-191.

Scatchard, G., 1949. The attraction of proteins for small molecules and ion. Ann. N.Y. Acad. Sci. 51, 660-672.

Schubiger, M., Tomita, S., Sung, C., Robinow, S., Truman, J.W., 2003. Isoform specific control of gene activity in vivo by the Drosophila ecdysone receptor. Mech. Dev. 120, 909-918.

Takimoto, G.S., Tung, L., Abdel-Hafiz, H., Abel, M.G., Sartorius, C.A., Richer, J.K., Jacobsen, B.M., Bain, D.L., Horwitz, K.B., 2003. Functional properties of the Nterminal region of progesterone receptors and their mechanistic relationship to structure. J Steroid Biochem. Mol. Biol. 85, 209-219.

Tetel, M.J., Giangrande, P.H., Leonhardt, S.A., McDonnell, D.P., Edwards, D.P., 1999. Hormone-dependent interaction between the amino- and carboxyl-terminal domains of progesterone receptor in vitro and in vivo. Mol. Endocrinol. 13, 910-924. 
Tian, H., Mahajan, M.A., Wong, C.T, Habeos, I., Samuels, H.H., 2006. The NTerminal A/B domain of the thyroid hormone receptor-beta2 isoform influences ligand-dependent recruitment of coactivators to the ligand-binding domain. Mol. Endocrinol. 20, 2036-2045.

Thomas, H.E., Stunnenberg, H.G., Stewart, A.F., 1993. Heterodimerization of the Drosophila ecdysone receptor with retinoid X receptor and ultraspiracle. Nature 362, 471-475.

Turberg, A., Spindler, K.-D., 1992. Properties of nuclear and cytosolic ecdysteroid receptors from an epithelial cell line from Chironomus tentans. J. Insect Physiol. 38, 82-91.

Verras, M., Gourzi, P., Zacharopoulou, A., Mintzas, A.C., 2002. Developmental profiles and ecdysone regulation of the mRNAs for two ecdysone receptor isoforms in the Mediterranean fruit fly Ceratitis capitata. Insect Mol. Biol. 11, 553-65.

Yao, T.P. Segraves, W.A., Oro, A.E., Mc Keown, M., Evans, R.M., 1992. Drosophila ultraspiracle modulates ecdysone receptor function via heterodimer formation. Cell $71,63-72$. 
Table

Table 1: Affinity of Ponasterone A to EcR/Usp heterodimers and receptor concentration in the absence of DNA.

\begin{tabular}{|c|c|c|}
\hline Receptors & $\begin{array}{c}K_{D}(n M)^{*} \\
M \pm S D, n=4-6\end{array}$ & 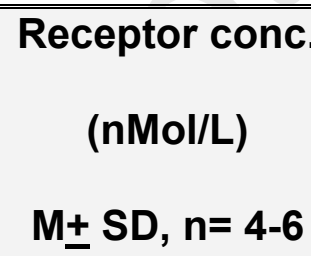 \\
\hline EcRA / Usp wt & $7.0 \pm 4.0$ & $0.17 \pm 0.02$ \\
\hline EcRB1 / Usp wt & $7.1 \pm 4.3$ & $0.21 \pm 0.11$ \\
\hline EcRB2 / Usp wt & $4.4 \pm 2.3$ & $0.16 \pm 0.04$ \\
\hline EcRA / Usp I & $6.6 \pm 1.1$ & $0.51 \pm 0.23$ \\
\hline EcRB1 / Usp I & $8.4 \pm 4.1$ & $0.16 \pm 0.16$ \\
\hline EcRB2 / Usp I & $8.9 \pm 1.8$ & $0.49 \pm 0.12$ \\
\hline EcRA / Usp II & $\overline{10.7 \pm 1.8}$ & $0.81 \pm 0.64$ \\
\hline EcRB1 / Usp II & $6.8 \pm 1.7$ & $0.39 \pm 0.25$ \\
\hline EcRB2 / Usp II & $11.0 \pm 1.5$ & $0.68 \pm 0.58$ \\
\hline EcRA / Usp III & $6.6 \pm 3.2$ & $0.27 \pm 0.14$ \\
\hline EcRB1 / Usp III & $6.5 \pm 4.1$ & $0.96 \pm 0.55$ \\
\hline EcRB2 / Usp III & $7.8 \pm 0.6$ & $0.74 \pm 0.30$ \\
\hline
\end{tabular}

${ }^{*}$ No significant differences are found for all combinations of EcR isoforms and Usp variants. 


\section{Figure}

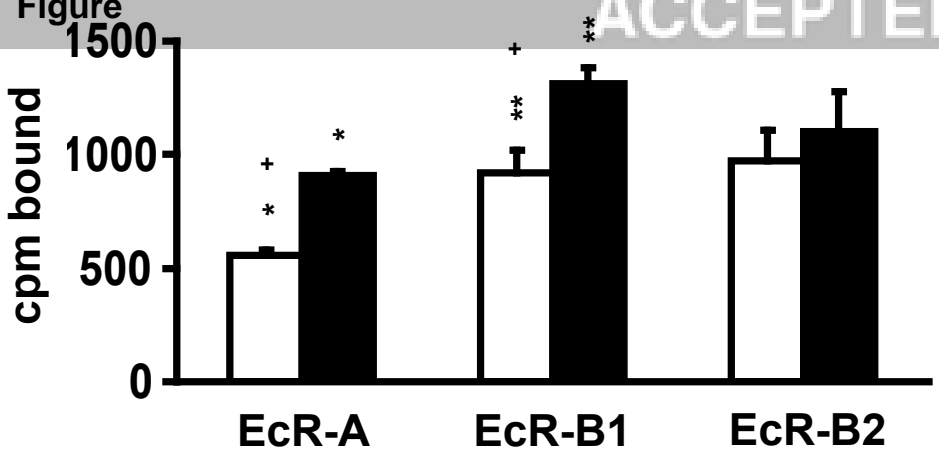

EcR-A

EcR-B1

EcR-B2 
Figurer

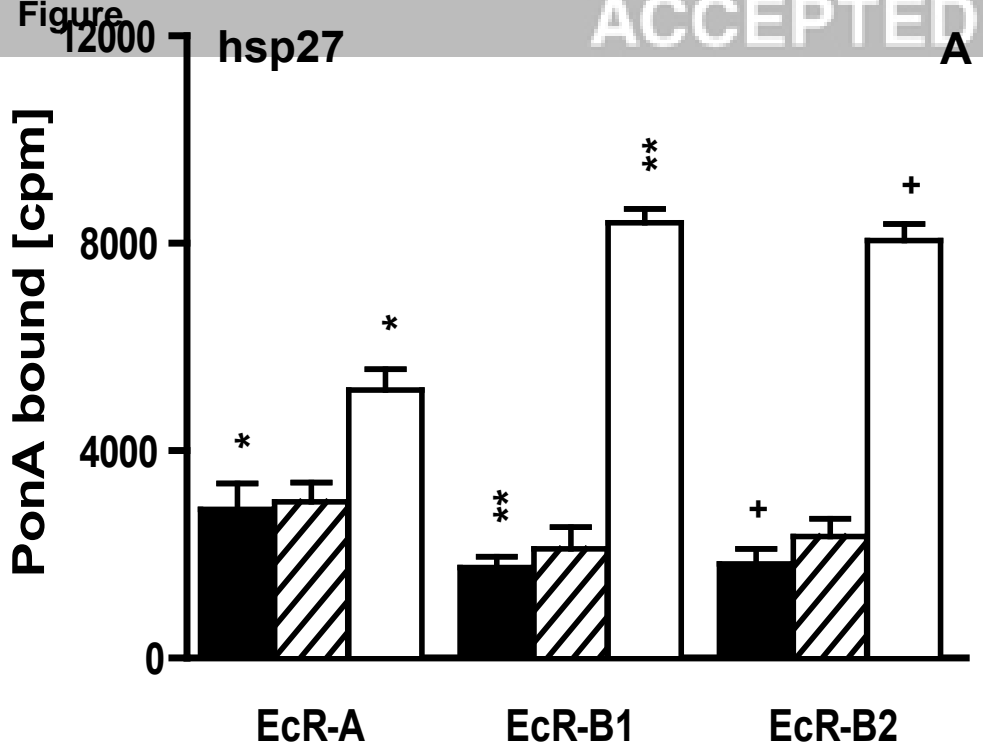

Fig.: 5
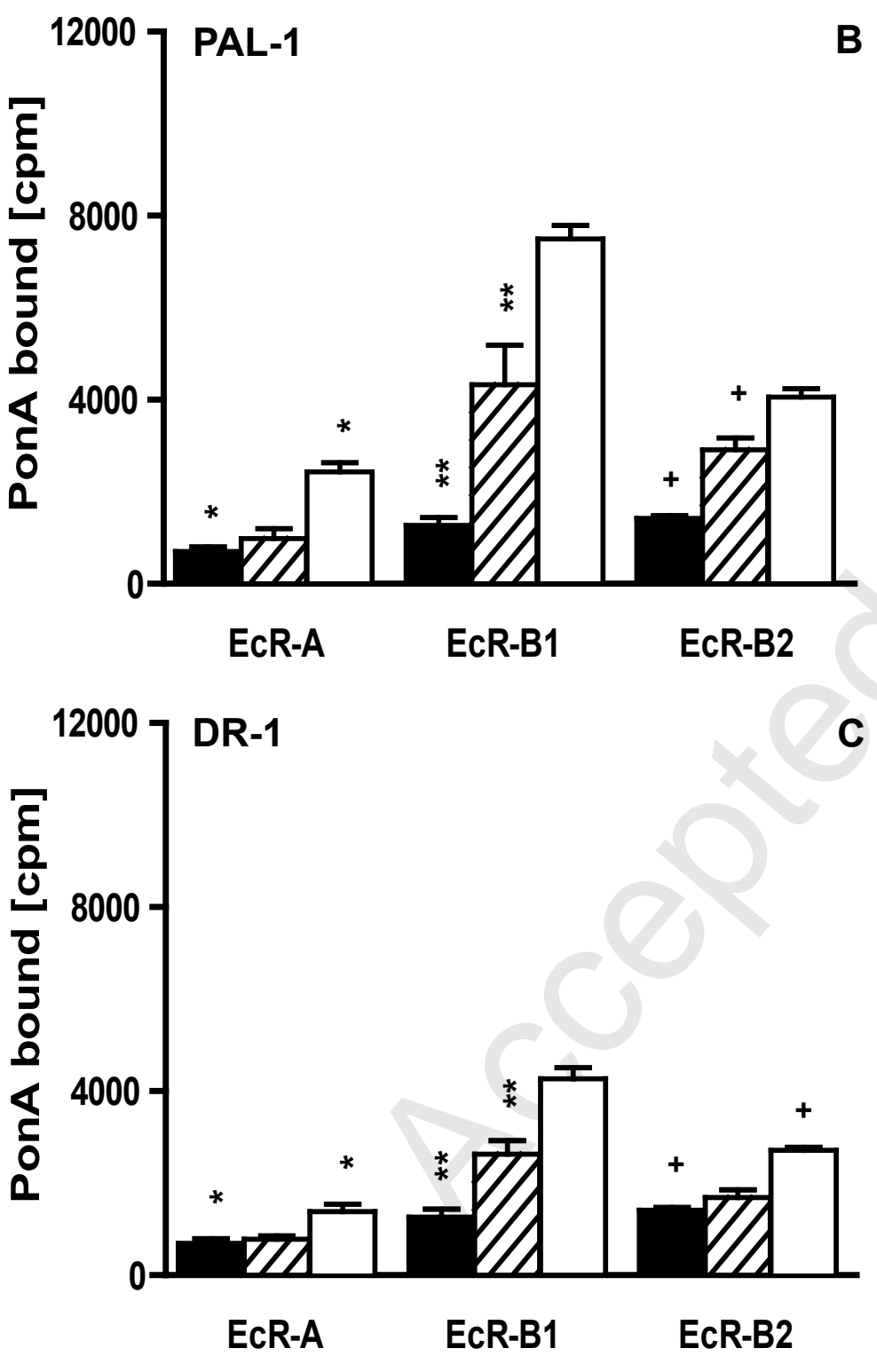

B

C 
Fig. $7 a$

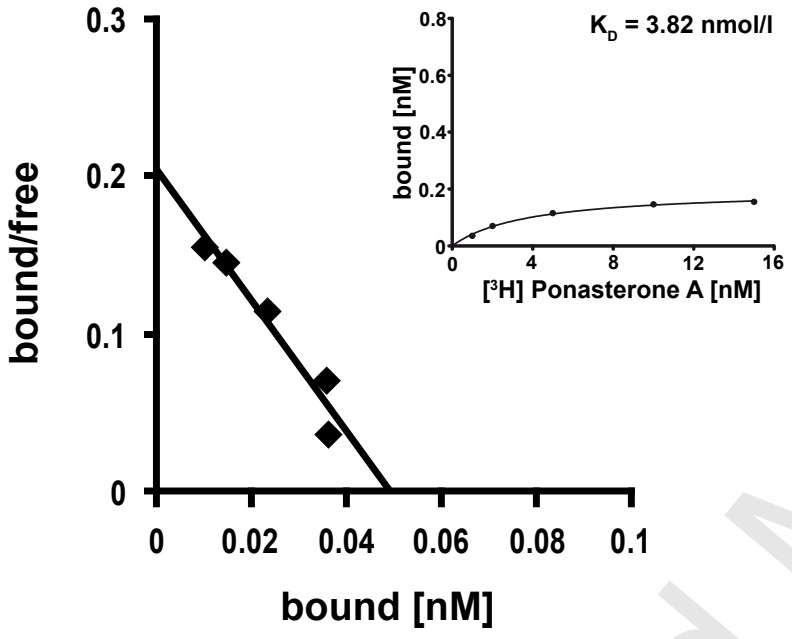




\section{Fig. 7b}

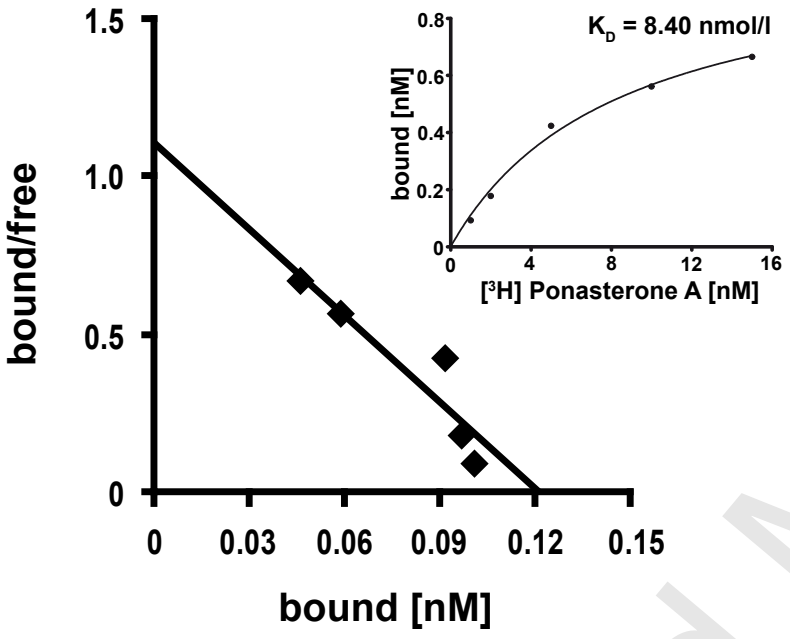


ఖ

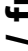

을

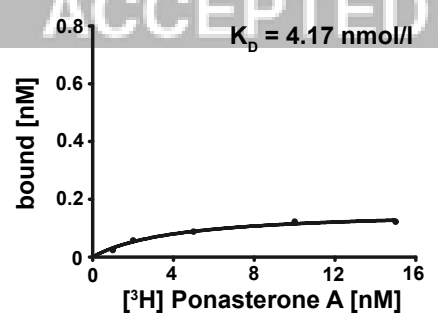

0.2

0.1

0

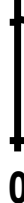

$0 \quad 0.1$

0.2

0.3

0.4

0.5

bound [nM]

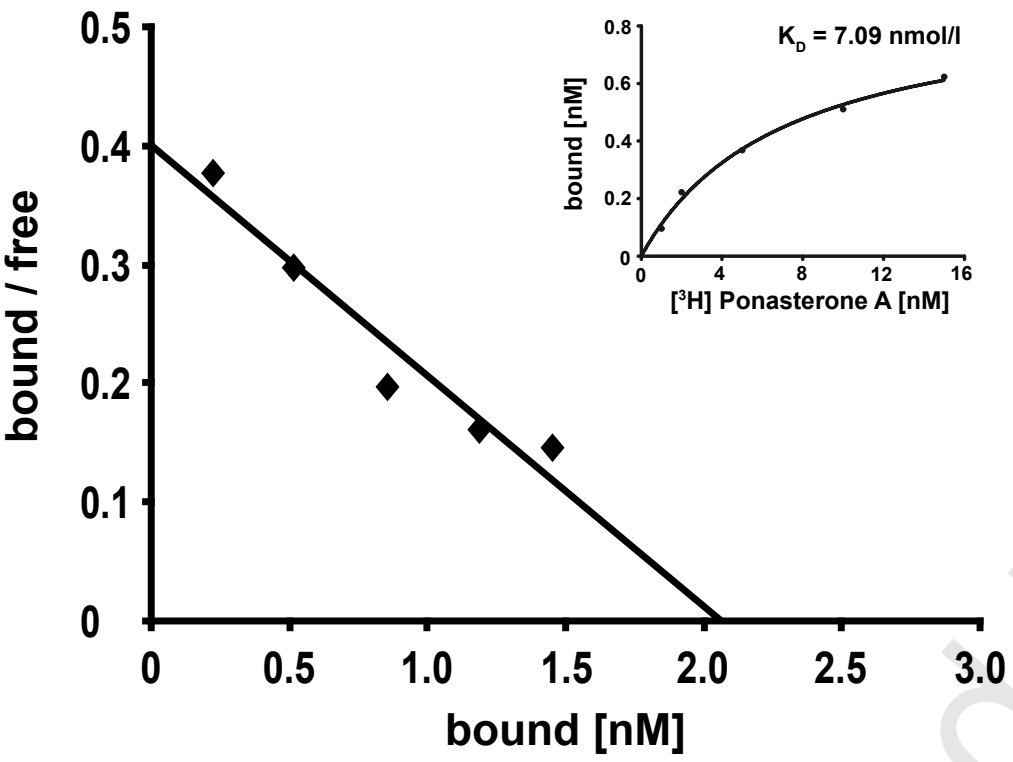

B 
